\title{
On Sheffer polynomial families
}

\author{
Sandra Pinelas ${ }^{1}$ and Paolo Emilio Ricci ${ }^{2, *}$ \\ ${ }^{1}$ Academia Militar - Departamento de Ciências Exactas e Engenharia, Av. Conde Castro Guimarães, 2720-113, Amadora, Portugal \\ ${ }^{2}$ International Telematic University UniNettuno, Corso Vittorio Emanuele II, 39, 00186, Roma, Italia
}

Received 16 October 2018, Accepted 22 February 2019

\begin{abstract}
Attention is focused to particular families of Sheffer polynomials which are different from the classical ones because they satisfy non-standard differential equations, including some of fractional type. In particular Sheffer polynomial families are considered whose characteristic elements are based on powers or exponential functions.
\end{abstract}

Keywords: Sheffer polynomial families, Generating functions, Monomiality principle, Fractional derivative equations, Combinatorial analysis

\section{Introduction}

In recent articles [1, 2], new sets of Sheffer [3] and Brenke [4] polynomials, based on higher order Bell numbers [5-9], have been studied. Furthermore, several integer sequences [10] associated with the considered polynomials sets both of exponential $[11,12]$ and logarithmic type have been introduced [2].

We recall that the exponential and logarithmic polynomials have been recently studied even in the multidimensional case $[13-15]$.

It is worth to note that the Sheffer family includes a plenty of unusual polynomials, which satisfy non-standard differential equations. In this article we focus our attention on Sheffer polynomial families whose characteristic elements are based on powers or exponential functions, deriving the relevant differential equations, which are frequently of fractional type.

\section{Sheffer polynomials}

We start recalling the particular meaning of the term set in the framework of polynomial theory.

Definition 2.1. A polynomial family $\left\{P_{n}(x)\right\}_{(n \geq 0)}$ is called a polynomial set iff $\forall n$, $\operatorname{deg} P_{n}=n$. In what follows, we are dealing with polynomial families that, in several cases, don't satisfy the above condition. The Sheffer polynomial families $\left\{s_{n}(x)\right\}$ are introduced [3] by means of the exponential generating function [16] of the type:

$$
A(t) \exp (x H(t))=\sum_{n=0}^{\infty} s_{n}(x) \frac{t^{n}}{n !}
$$

where,

$$
\begin{aligned}
& A(t)=\sum_{n=0}^{\infty} a_{n} \frac{t^{n}}{n !}, \quad\left(a_{0} \neq 0\right), \\
& H(t)=\sum_{n=0}^{\infty} h_{n} \frac{t^{n}}{n !}, \quad\left(h_{0}=0\right) .
\end{aligned}
$$

Remark 2.2. It is well known $[4,17]$ that there is a natural link between the function $H(t)$ and the degree of polynomials $s_{n}(x)$ in expansion (1). Namely,

$$
\operatorname{deg} s_{n}=n i f f, \quad \text { in equation } \quad(2), \quad h_{1} \neq 0 .
$$

*Corresponding author: paoloemilioricci@gmail.com 
Actually, in what follows, if $H(t)$ is a polynomial of degree $m$, we have found that $\operatorname{deg} s_{n} \leq\left[\frac{n}{m}\right]$, where [.] denotes the integral part.

In general, we are dealing with a Sheffer polynomial set iff the condition $h_{1} \neq 0$ is satisfied.

According to a different characterization (see [18], p. 18), the same polynomial sequence can be defined by means of the pair $(g(t), f(t))$, where $g(t)$ is an invertible series and $f(t)$ is a delta series:

$$
\begin{gathered}
g(t)=\sum_{n=0}^{\infty} g_{n} \frac{t^{n}}{n !}, \quad\left(g_{0} \neq 0\right), \\
f(t)=\sum_{n=0}^{\infty} f_{n} \frac{t^{n}}{n !}, \quad\left(f_{0}=0, f_{1} \neq 0\right) .
\end{gathered}
$$

Denoting by $f^{-1}(t)$ the compositional inverse of $f(t)$ (i.e., such that $\left.f\left(f^{-1}\right)(t)\right)=\left(f^{-1}\right)(f(t))=t$ ), the exponential generating function of the sequence $\left\{s_{n}(x)\right\}$ is given by

$$
\frac{1}{g\left[f^{-1}(t)\right]} \exp \left(x f^{-1}(t)\right)=\sum_{n=0}^{\infty} s_{n}(x) \frac{t^{n}}{n !}
$$

so that,

$$
A(t)=\frac{1}{g\left[f^{-1}(t)\right]}, \quad H(t)=f^{-1}(t)
$$

When $g(t) \equiv 1$, the Sheffer sequence corresponding to the pair $(1, f(t))$ is called the associated Sheffer sequence $\left\{\sigma_{n}(x)\right\}$ for $f(t)$, and its exponential generating function is given by

$$
\exp \left(x f^{-1}(t)\right)=\sum_{n=0}^{\infty} \sigma_{n}(x) \frac{t^{n}}{n !} .
$$

A list of known Sheffer polynomial sequences and their associated ones can be found in [19]. New Euler-type Sheffer polynomials have been recently introduced in [20].

\section{Power based Sheffer polynomials}

In this section we derive Sheffer polynomial families assuming the following basic functions:

$$
\frac{A^{\prime}(t)}{A(t)}=t^{p}, \quad H(t)=t^{q},
$$

$p$ and $q$ positive integer numbers, so that,

$$
A(t)=\exp \left(\frac{t^{p+1}}{p+1}\right)
$$

and the generating function is:

$$
G(t, x)=\exp \left(\frac{t^{p+1}}{p+1}+x t^{q}\right)=\sum_{n=0}^{\infty} \tilde{q}_{n}(p ; q ; x) \frac{t^{n}}{n !}
$$

Therefore, denoting by $H^{-1}(t)$ the compositional inverse of $H(t)$, we have:

$$
H^{-1}(t)=t^{1 / q}, \quad H^{\prime}(t)=q t^{q-1}
$$

Definition 3.1. We recall that a polynomial set $\left\{p_{n}(x)\right\}$ is called quasi-monomial if and only if there exist two operators $\hat{P}$ and $\hat{M}$ such that,

$$
\begin{gathered}
\hat{P}\left(p_{n}(x)\right)=n p_{n-1}(x), \\
\hat{M}\left(p_{n}(x)\right)=p_{n+1}(x), \quad(n=1,2, \ldots) .
\end{gathered}
$$

$\hat{P}$ is called the derivative operator and $\hat{M}$ the multiplication operator, as they act in the same way of classical operators on monomials. 
This definition traces back to a paper by J.F. Steffensen [21], recently improved by G. Dattoli [22] and widely used in several applications (see e.g., [23, 24] and the references therein).

Y. Ben Cheikh [25] proved that every polynomial set is quasi-monomial under the action of suitable derivative and multiplication operators. In particular, if the considered polynomial set is Sheffer, the Corollary 3.2 in the same article ensure that the derivative and multiplication operator are given by:

$$
\begin{gathered}
\hat{P}=H^{-1}\left(D_{x}\right), \\
\hat{M}=\frac{A^{\prime}\left[H^{-1}\left(D_{x}\right)\right]}{A\left[H^{-1}\left(D_{x}\right)\right]}+x H^{\prime}\left[H^{-1}\left(D_{x}\right)\right],
\end{gathered}
$$

where prime denotes the derivative with respect to $t$, and $D_{x}$ the derivative with respect to $x$.

Remark 3.2. It is worth to note that the above mentioned result (Corollary 3.2 in [25]), given for polynomial sets, never uses in proof the condition $h_{1} \neq 0$. Therefore, it can be applied even to polynomials defined by Sheffer generating functions (1), i.e. to Sheffer polynomial families.

According to the above equations (8), (10), (12), we have the result:

Theorem 3.3. The derivative and multiplication operators of the Sheffer polynomial family defined by the generating function (9) are given by,

$$
\begin{gathered}
\hat{P}=D_{x}^{1 / q}, \\
\hat{M}=D_{x}^{p / q}+q x D_{x}^{(q-1) / q} .
\end{gathered}
$$

\subsection{Differential equation}

As a consequence of the monomiality principle, the factorization method gives the differential equation satisfied by the quasi-monomial polynomials $\left\{p_{n}(x)\right\}$ in the form:

$$
\hat{M} \hat{P} p_{n}(x)=n p_{n}(x) .
$$

In the present case, we have the result:

Theorem 3.5. The Sheffer polynomials $\left\{\tilde{q}_{n}(p, q ; x)\right\}$ satisfy the differential equation

$$
\left(D_{x}^{(p+1) / q}+q x D_{x}\right) \tilde{q}_{n}(p, q ; x)=n \tilde{q}_{n}(p, q ; x) .
$$

\section{Some particular examples}

We show in this Section some particular example.

\subsection{Case $p=3, q=2, \quad G(t, x)=\exp \left[\mathrm{t}^{\frac{4}{4}}+\mathrm{xt}^{2}\right]$}

The ordinary differential equation is:

$$
\left(D_{x}^{2}+2 x D_{x}\right) \tilde{q}_{n}(3,2 ; x)=n \tilde{q}_{n}(3,2 ; x) .
$$

The first few $\tilde{q}_{n}(3,2 ; x)$ polynomials are as follows:

$$
\begin{aligned}
& \tilde{q}_{0}(3,2 ; x)=1, \\
& \tilde{q}_{2 k+1}(3,2 ; x)=0, \quad \forall k \geq 0, \\
& \tilde{q}_{2}(3,2 ; x)=2 x, \\
& \tilde{q}_{4}(3,2 ; x)=6\left(2 x^{2}+1\right), \\
& \tilde{q}_{6}(3,2 ; x)=180\left(4 x^{3}+x\right), \\
& \tilde{q}_{8}(3,2 ; x)=420\left(4 x^{4}+12 x^{2}+3\right) .
\end{aligned}
$$

Further values can be easily achieved by using Wolfram Alpha ${ }^{\odot}$. 
Remark 4.1. Note that there is a link of the $\tilde{q}_{n}(3,2 ; x)$ polynomials with the classical $H_{n}(x)$. In fact, let

$$
\begin{gathered}
G_{1}(x, t)=\exp \left(2 x \tau-\tau^{2}\right)=\sum_{n=0}^{\infty} H_{n}(\tau) \frac{\tau^{n}}{n !} \\
G_{2}(x, t)=\exp \left(x t^{2}+t^{4} / 4\right)=\sum_{n=0}^{\infty} \tilde{q}_{n}(3,2 ; x) \frac{t^{n}}{n !} .
\end{gathered}
$$

Since $G_{2}(x,-t)=G_{2}(x, t)$, it follows that $q_{2 k+1}(3,2 ; x)=0, \forall k \geq 0$, and

$$
G_{2}(x, t)=\sum_{n=0}^{\infty} \tilde{q}_{2 n}(3,2 ; x) \frac{t^{2 n}}{2 n !} .
$$

Therefore, putting $t^{2}=2$ is (i the imaginary unit), we find,

$$
G_{1}(\mathrm{i} x, s)=\exp \left(2 \mathrm{i} x s-\mathrm{s}^{2}\right) \sum_{n=0}^{\infty}(2 \mathrm{i})^{n} \tilde{q}_{2 n}(3,2 ; x) \frac{s^{n}}{2 n !},
$$

and consequently,

$$
\tilde{q}_{2 n}(3,2 ; x)=\frac{(2 n) !}{2^{n} n !} \frac{H_{n}(\mathrm{i} x)}{\mathrm{i}^{n}}=(2 n-1) ! ! \frac{H_{n}(\mathrm{i} x)}{\mathrm{i}^{n}} .
$$

In a similar way, when $\frac{p+1}{q}=N,(N$ integer number $)$, we can find a link between the Gould-Hopper polynomials defined by the generating function,

$$
G_{1}^{*}(x, t)=\exp \left(x \tau+\tau^{N}\right)=\sum_{n=0}^{\infty} H_{n}^{(N)}(\tau) \frac{\tau^{n}}{n !}
$$

and the Sheffer polynomials defined by,

In fact, owing the symmetry of these polynomials, we have,

$$
G_{2}^{*}(x, t)=\exp \left(x t+t^{(p+1) / q}\right)=\sum_{n=0}^{\infty} \tilde{q}_{n}(p, q ; x) \frac{t^{n}}{n !} .
$$

$$
\tilde{q}_{N n+k}(p, q ; x)=0, \quad \forall n \geq 0, k=1,2, \ldots, N-1,
$$

and therefore, putting $\tau-t^{N}$, we find:

$$
G_{2}^{*}(x, t)=\sum_{n=0}^{\infty} \tilde{q}_{N n}(p, q ; x) \frac{t^{N n}}{(N n) !}=\sum_{n=0}^{\infty} \tilde{q}_{n}(p, q ; x) \frac{\tau^{n}}{(N n) !},
$$

so that $\tilde{q}_{n}(p, q ; x)$, as a polynomial of degree $n$, can be expressed in terms of the Gould-Hopper polynomials by:

$$
\tilde{q}_{N n}(p, q ; x)=\frac{(N n) !}{n !} H_{n}^{(N)}(x) .
$$

\section{Remark 4.2.}

1. The equation (16) is an ordinary differential equation of order 2 , because $(p+1) / q=4 / 2=2$. This happens, in general, if and only if $\frac{p+1}{q}=N,(N$ integer number).

2. Note that, owing to the fractional derivative operator $\hat{P}=D_{x}^{1 / 2}$, the polynomial degree increases slowly, actually the degree of $\tilde{q}_{2 n}(p, q ; x)$ is equal to $(2 n) / 2=n$.

\subsection{Case $p=1, q=3, \quad G(t, x)=\exp \left[\frac{t^{2}}{2}+x t^{3}\right]$}

The fractional differential equation is:

$$
\left(D_{x}^{2 / 3}+3 x D_{x}\right) \tilde{q}_{n}(1,2 ; x)=n \tilde{q}_{n}(1,3 ; x) .
$$

The first few $\tilde{q}_{n}(1,3 ; x)$ polynomials are as follows:

If $n \equiv 0,(\bmod 3)$ :

$$
\begin{aligned}
& \tilde{q}_{0}(1,3 ; x)=1, \\
& \tilde{q}_{3}(1,3 ; x)=6 x, \\
& \tilde{q}_{6}(1,3 ; x)=15\left(24 x^{2}+1\right), \\
& \tilde{q}_{9}(1,3 ; x)=7560\left(8 x^{3}+x\right), \\
& \tilde{q}_{12}(1,3 ; x)=10395\left(1920 x^{4}+480 x^{2}+1\right) .
\end{aligned}
$$


If $n \equiv 1,(\bmod 3):$

$$
\begin{aligned}
& \tilde{q}_{1}(1,3 ; x)=0, \\
& \tilde{q}_{4}(1,3 ; x)=3, \\
& \tilde{q}_{7}(1,3 ; x)=630 x, \\
& \tilde{q}_{10}(1,3 ; x)=945\left(240 x^{2}+1\right), \\
& \tilde{q}_{13}(1,3 ; x)=1621620\left(80 x^{3}+x\right) .
\end{aligned}
$$

If $n \equiv 2,(\bmod 3):$

$$
\begin{aligned}
& \tilde{q}_{2}(1,3 ; x)=1, \\
& \tilde{q}_{5}(1,3 ; x)=60 x, \\
& \tilde{q}_{8}(1,3 ; x)=105\left(96 x^{2}+1\right), \\
& \tilde{q}_{11}(1,3 ; x)=103950\left(32 x^{3}+x\right), \\
& \tilde{q}_{14}(1,3 ; x)=135135\left(13440 x^{4}+840 x^{2}+1\right) .
\end{aligned}
$$

Further values can be easily achieved by using Wolfram Alpha ${ }^{\odot}$.

\section{Remark 4.3.}

1. The equation (17) is a fractional differential equation.

2. Note that, owing to the fractional derivative operator $\hat{P}=D_{x}^{1 / 3}$, the degree of the polynomial $\tilde{q}_{n}(x ; 3)$ is equal to:

- $n / 3$ if $n \equiv 0,(\bmod 3)$,

- $(n-4) / 3$ if $n \equiv 1,(\bmod 3)$, and $n \geq 4$,

- $(\mathrm{n}-2) / 3$ if $n \equiv 2,(\bmod 3)$, and $n \geq 2$.

\section{A particular family of Sheffer polynomials}

In this Section we consider a particular family of Sheffer polynomials defined by generating functions of the type:

$$
G(t, x)=A(t) \exp [x H(t)], \quad \text { where } \frac{A^{\prime}(t)}{A(t)}=H(t),
$$

and $H(t)$ is an invertible function. Therefore, we find,

$$
\begin{gathered}
A(t)=\exp \left[\int H(t) d t\right], \quad \text { where } \\
G(t, x)=\exp \left[\int H(t) d t+x H(t)\right]=\sum_{n=0}^{\infty} \tau_{n}(x) \frac{t^{n}}{n !} .
\end{gathered}
$$

Putting, as before, $f(t)=H^{-1}(t)$, according to the recalled result by Y. Ben Cheikh [25], the derivative and multiplication operators for the relevant polynomials $\tau_{n}$ are given by:

$$
\begin{gathered}
\hat{P}=H^{-1}\left(D_{x}\right), \\
\hat{M}=D_{x}+x H^{\prime}\left[H^{-1}\left(D_{x}\right)\right],
\end{gathered}
$$

so that we have the result:

Theorem 5.1. The Sheffer polynomials $\left\{\tau_{n}(x)\right\}$ satisfy the differential equation

$$
\left[D_{x}+x H^{\prime}\left(H^{-1}\left(D_{x}\right)\right)\right] H^{-1}\left(D_{x}\right) \tau_{n}(x)=n \tau_{n}(x) .
$$

\section{Particular examples}

Note that the particular Sheffer polynomials of Section 5 only depend on $H(t)$. 


\subsection{A power based example, linked to Section 3}

We assume,

$$
H(t)=\frac{t^{2 q+1}}{2 q+1}
$$

( $q$ a positive integer number), so that,

$$
\begin{gathered}
H^{\prime}(t)=t^{2 q}, \quad H^{-1}(t)=[(2 q+1) t]^{1 /(2 q+1)} \\
G(t, x)=\exp \left[\frac{1}{2 q+1}\left(\frac{1}{2 q+2} t^{2 q+2}+x t^{2 q+1}\right)\right]=\sum_{n=0}^{\infty} \tilde{\tau}_{n}(x ; q) \frac{t^{n}}{n !} .
\end{gathered}
$$

According to the above results, the derivative and multiplication operators for the quasi-monomials $\tilde{\tau}_{n}(x ; q)$ are given by,

$$
\begin{gathered}
\hat{P}=\left[(2 q+1) D_{x}\right]^{1 /(2 q+1)}, \\
\hat{M}=D_{x}+x\left[(2 q+1) D_{x}\right]^{2 q /(2 q+1)},
\end{gathered}
$$

and the relevant differential equation writes,

$$
\left[D_{x}+x\left[(2 q+1) D_{x}\right]^{2 q /(2 q+1)}\right]\left[(2 q+1) D_{x}\right]^{1 /(2 q+1)} \tilde{\tau}_{n}(x ; q)=n \tilde{\tau}_{n}(x ; q)
$$

that is,

$$
(2 q+1)\left[D_{x}^{(2 q+2) /(2 q+1)}+x D_{x}\right] \tilde{\tau}_{n}(x ; q)=n \tilde{\tau}_{n}(x ; q)
$$

which is a fractional derivative equation.

6.2 Case $q=1, \quad H(t)=t^{3} / 3$

The generating function is,

$$
G(t, x)=\exp \left[\frac{1}{3}\left(\frac{1}{4} t^{4}+x t^{3}\right)\right]=\sum_{n=0}^{\infty} \tilde{\tau}_{n}(x ; 3) \frac{t^{n}}{n !}
$$

and the fractional differential equation (25) writes,

$$
3\left[D_{x}^{4 / 3}+x D_{x}\right] \tilde{\tau}_{n}(x ; 3)=n \tilde{\tau}_{n}(x ; 3) .
$$

The first few $\tilde{\tau}_{n}(x ; 3)$ polynomials are as follows:

$$
\begin{array}{lll}
\tilde{\tau}_{0}(x ; 3)=1, & \tilde{\tau}_{1}(x ; 3)=0, & \tilde{\tau}_{2}(x ; 3)=0, \\
\tilde{\tau}_{3}(x ; 3)=2 x, & \tilde{\tau}_{4}(x ; 3)=2, & \tilde{\tau}_{5}(x ; 3)=0, \\
\tilde{\tau}_{6}(x ; 3)=40 x^{2}, & \tilde{\tau}_{7}(x ; 3)=140 x, & \tilde{\tau}_{8}(x ; 3)=140, \\
\tilde{\tau}_{9}(x ; 3)=2240 x^{3}, & \tilde{\tau}_{10}(x ; 3)=16800 x^{2}, & \tilde{\tau}_{11}(x ; 3)=46200 x, \\
\tilde{\tau}_{12}(x ; 3)=15400\left(16 x^{4}+3\right), & \tilde{\tau}_{13}(x ; 3)=3203200 x^{3}, & \tilde{\tau}_{14}(x ; 3)=16816800 x^{2} .
\end{array}
$$

Further values can be easily achieved by using Wolfram Alpha ${ }^{\circledR}$.

Note the symmetry of the above scheme, according to which the degree of the polynomial $\tilde{\tau}_{n}(x ; 3)$ is equal to:

- $n / 3$ if $n \equiv 0,(\bmod 3)$,

- $(n-4) / 3$ if $n \equiv 1,(\bmod 3)$, and $n \geq 4$,

- $(n-8) / 3$ if $n \equiv 2,(\bmod 3)$, and $n \geq 8$.

\subsection{An exponential based example}

We assume,

$$
H(t)=e^{t}-1,
$$


so that,

$$
\begin{gathered}
H^{\prime}(t)=e^{t}, \quad H^{-1}(t)=\log (t+1) \\
G(t, x)=\exp \left[e^{t}-t+x\left(e^{t}-1\right)\right]=\sum_{n=0}^{\infty} \tilde{\sigma}_{n}(x) \frac{t^{n}}{n !} .
\end{gathered}
$$

According to the above results, the derivative and multiplication operators for the quasi-monomials $\tilde{\sigma}_{n}(x)$ are given by,

$$
\begin{gathered}
\hat{P}=\log \left(D_{x}+1\right), \\
\hat{M}=D_{x}+x\left(D_{x}+1\right),
\end{gathered}
$$

and the relevant differential equation writes,

$$
\left[D_{x}+x\left(D_{x}+1\right)\right] \log \left(D_{x}+1\right) \tilde{\sigma}(x)=n \tilde{\sigma}(x),
$$

that is,

$$
\left[D_{x}+x\left(D_{x}+1\right)\right] \sum_{k=1}^{n}(-1)^{k+1} \frac{D_{x}^{k}}{k} \tilde{\sigma}_{n}(x)=n \tilde{\sigma}_{n}(x),
$$

which is an infinite order differential equation reducing to an equation of order $n$ when applied to a polynomial of degree $n$. The first few $\tilde{\sigma}_{n}(x)$ polynomials are as follows:

$$
\begin{aligned}
& \tilde{\sigma}_{0}(x)=1 \\
& \tilde{\sigma}_{1}(x)=x \\
& \tilde{\sigma}_{2}(x)=x^{2}+x+1 \\
& \tilde{\sigma}_{3}(x)=x^{3}+3 x^{2}+4 x+1 \\
& \tilde{\sigma}_{4}(x)=x^{4}+6 x^{3}+13 x^{2}+11 x+4 \\
& \tilde{\sigma}_{5}(x)=x^{5}+10 x^{4}+35 x^{3}+55 x^{2}+41 x+11 \\
& \tilde{\sigma}_{6}(x)=x^{6}+15 x^{5}+80 x^{4}+200 x^{2}+256 x^{2}+162 x+41 \\
& \tilde{\sigma}_{7}(x)=x^{7}+21 x^{6}+161 x^{5}+595 x^{4}+1176 x^{3}+1274 x^{2}+715 x+162, \\
& \tilde{\sigma}_{8}(x)=x^{8}+28 x^{7}+294 x^{6}+1526 x^{5}+4361 x^{4}+7182 x^{3}+6791 x^{2}+3425 x+715 .
\end{aligned}
$$

Further values can be easily achieved by using Wolfram Alpha ${ }^{\circledR}$.

Remark 6.1. Note that the sequence:

$$
1,1,3,9,35,153,755,4105,24323, \ldots,
$$

(that is the values $\tilde{\sigma}_{n}(1)$ ), has a combinatorial character, since it appears in the Encyclopedia of integer sequences under A217924 - Row sequence of table A217537, $a(n):=\sum_{j=0}^{k}\left(\sum_{k=0}^{n} n_{k-j} 2^{j}(-1)^{(k-j)} \operatorname{Stirling}_{2}(n-k+j, j)\right) ;-\operatorname{Vladimir}$ Kruchinin, Feb 28, 2015.

Furthermore, the sequence:

$$
1,1,4,11,41,162,715, \ldots,
$$

(that is the values $\tilde{\sigma}_{n}(0), n \geq 2$ ) appears in the Encyclopedia of integer sequences under A000296 - Set partitions without singletons: number of partitions of an $n$-set into blocks of size $>1$. Also number of cyclically spaced (or feasible) partitions.

\section{A mixed-type (power-exp) Sheffer polynomial family}

We assume in this Section:

$$
H(t)=t^{q}, \quad A(t)=\exp \left(e^{t}-1\right)
$$

( $q$ a positive integer number), so that,

$$
\begin{gathered}
H^{\prime}(t)=q t^{q-1}, \quad H^{-1}(t)=t^{1 / q}, \\
G(t, x)=\exp \left[e^{t}-1+x t^{q}\right]=\sum_{n=0}^{\infty} \tilde{\rho}_{n}(x ; q) \frac{t^{n}}{n !} .
\end{gathered}
$$


According to the above results, the derivative and multiplication operators for the quasi-monomials $\tilde{\rho}_{n}(x ; q)$ are given by,

$$
\begin{gathered}
\hat{P}=D_{x}^{1 / q}, \\
\hat{M}=\exp \left(D_{x}^{1 / q}\right)+q x D_{x}^{(q-1) / q},
\end{gathered}
$$

and the relevant differential equation writes,

$$
\left[\exp \left(D_{x}^{1 / q}\right) D_{x}^{1 / q}+q x D_{x}\right] \tilde{\rho}_{n}(x ; q)=n \tilde{\rho}_{n}(x ; q),
$$

that is,

$$
\left[\sum_{k=0}^{q n-1} \frac{D_{x}^{(k+1) / q}}{k !}+q x D_{x}\right] \tilde{\rho}_{n}(x ; q)=n \tilde{\rho}_{n}(x ; q),
$$

which is an infinite order differential equation reducing to an equation of order $q n-1$ when it is applied to a polynomial of degree $n$.

\subsection{Case $q=2, \quad \mathrm{G}(t, x)=\exp \left[e^{t}-1+x t^{2}\right]$}

The first few $\tilde{\rho}_{n}(x ; 2)$ polynomials are as follows:

$$
\begin{aligned}
& \tilde{\rho}_{0}(x ; 2)=1, \\
& \tilde{\rho}_{1}(x ; 2)=1 \\
& \tilde{\rho}_{2}(x ; 2)=2 x+2 \\
& \tilde{\rho}_{3}(x ; 2)=6 x+5 \\
& \tilde{\rho}_{4}(x ; 2)=12 x^{2}+24 x+15 \\
& \tilde{\rho}_{5}(x ; 2)=60 x^{2}+50 x+52 \\
& \tilde{\rho}_{6}(x ; 2)=120 x^{3}+360 x^{3}+450 x+203, \\
& \tilde{\rho}_{7}(x ; 2)=840 x^{3}+2100 x^{2}+2184 x+877, \\
& \tilde{\rho}_{8}(x ; 2)=1680 x^{4}+6720 x^{3}+2520 x^{3}+11368 x+4140 .
\end{aligned}
$$

Further values can be easily achieved by using Wolfram Alpha ${ }^{\odot}$.

\section{Remark 7.1.}

1. Note that owing to the fractional derivative operator $\hat{P}=D_{x}^{1 / 2}$, the degree of the polynomial $\tilde{\rho}_{n}(x ; 2)$ is equal to $\left[\frac{n}{2}\right]$.

A similar phenomenon also applies to $q>2$.

2. Note that the sequence:

$$
1,1,2,5,15,52,203,877,4140, \ldots,
$$

(that is the values $\tilde{\rho}_{n}(0 ; 2)$ ), has a combinatorial character, since it appears in the Encyclopedia of integer sequences under A000110 - Bell or exponential numbers: number of ways to partition a set of $n$ labeled elements.

\section{Conclusion}

We have introduced "unusual families" of Sheffer polynomials, namely they satisfy non-standard differential equations, including some of fractional type.

In some case, we have noticed connections with particular integer sequences, since the polynomial - in suitable points exhibit a combinatorial character. In this article have considered Sheffer polynomials based on powers or exponential functions. In subsequent papers further families will be introduced, by using different basic elements.

\section{Author contributions}

The authors claim to have contributed equally and significantly in this paper. Both authors read and approved the final manuscript. 


\section{Acknowledgments}

The authors thanks the anonymous referee for his skillful remarks, useful for improving the manuscript.

\section{Compliance with ethical standards \\ Conflict of interest}

The authors declare that they have not received funds from any institution and that they have no conflict of interest.

\section{References}

1. Ricci PE, Natalini P, Bretti G (2017), Sheffer and Brenke polynomials associated with generalized Bell numbers. Jnanabha, Vijnana Parishad of India 47, 2, 337-352.

2. Bretti G, Natalini P, Ricci PE (2019), A new set of Sheffer-Bell polynomials and logarithmic numbers. Georgian Math J, https://doi.org/10.1515/gmj-2019-2007.

3. Sheffer IM (1939), Some properties of polynomials sets of zero type. Duke Math J 5, 590-622.

4. Brenke WC (1945), On generating functions of polynomial systems. Am Math Mon 52, 297-301.

5. Natalini P, Ricci PE (2004), An extension of the Bell polynomials. Comput Math Appl 47, 719-725.

6. Bernardini A, Natalini P, Ricci PE (2005), Multi-dimensional Bell polynomials of higher order. Comput Math Appl 50, $1697-1708$.

7. Natalini P, Ricci PE (2016), Remarks on Bell and higher order Bell polynomials and numbers. Cogent Math 3, 1-15.

8. Natalini P, Ricci PE (2017), Higher order Bell polynomials and the relevant integer sequences. Appl Anal Discrete Math 11, $327-339$.

9. Natalini P, Ricci PE (2018), Bell-Sheffer polynomial sets. Axioms 7, 71. https://doi.org/10.3390/axioms7040071.

10. Sloane NJA (2016), On-line encyclopedia of integer sequences, Published electronically at http://oeis.org.

11. Bell ET (1934), Exponential polynomials. Ann Math 35, 258-277.

12. Bell ET (1938), The iterated exponential integers. Ann Math 39, 539-557.

13. Feng Q, Da-Wei N, Dongkyu L, Bai-Ni G (2018), Some properties and an application of multivariate exponential polynomials, HAL archives, available online at https://hal.archives-ouvertes.fr/hal-01745173.

14. Feng Q (2018), Integral representations for multivariate logarithmic potentials. J Comput Appl Math 336, 54-62.

15. Feng Q (2018), On multivariate logarithmic polynomials and their properties. Indag Math 29, 5, $1179-1192$.

16. Srivastava HM, Manocha HL (1984), A treatise on generating functions, Halsted Press (Ellis Horwood Limited, Chichester), John Wiley and Sons, New York, Chichester, Brisbane and Toronto.

17. Huff WN (1947), The type of the polynomials generated by $f(x t) \phi(t)$. Duke Math J 14, 4, 1091-1104.

18. Roman SM (1984), The umbral calculus, Academic Press, New York.

19. Boas RP, Buck RC (1958), Polynomial expansions of analytic functions, Springer-Verlag, Berlin, Gottingen, Heidelberg, New York.

20. Bretti G, Natalini P, Ricci PE (2018), New sets of Euler-type polynomials. J Ana Num Theor 6, 2, 51-54.

21. Steffensen JF (1941), The poweroid, an extension of the mathematical notion of power. Acta Math 73, 333-366.

22. Dattoli G (2000), Hermite-Bessel and Laguerre-Bessel functions: a by-product of the monomiality principle, in: Cocolicchio D, Dattoli G, Srivastava HM (Eds.), Advanced Special Functions and Applications (Proceedings of the Melfi School on Advanced Topics in Mathematics and Physics; Melf, 9-12 May, 1999), Aracne, Roma, pp. 147-164.

23. Dattoli G, Ricci PE, Srivastava HM (Eds.) (2003), Advanced special functions and related topics in probability and in differential equations (Proceedings of the Melfi school on advanced topics in mathematics and Physics; Melfi, June 24-29, 2001). Appl Math Comput 141, 1, 1-230.

24. Dattoli G, Germano B, Martinelli MR, Ricci PE (2009), Monomiality and partial differential equations. Math Comput Model 50, 1332-1337.

25. Ben Cheikh Y (2003), Some results on quasi-monomiality. Appl Math Comput 141, 63-76.

Cite this article as: Pinelas S, Ricci P.E 2019. On Sheffer polynomial families. 4open, 2, 4. 